\title{
Numerical solution of singularly perturbed self-adjoint boundary value problem using Galerkin method
}

\author{
Murad Hussein Salih ${ }^{1}$, Gemechis File Duressa ${ }^{2}$, Habtamu Garoma Debela ${ }^{3 *}$ \\ ${ }_{1,2,3^{*}}$ Department of Mathematics, Jimma University, Jimma, ETHIOPIA \\ ${ }^{*}$ Corresponding Author: e-mail:habte200@ gmail.com, Tel: +251923432687 \\ ORCID iD: https://orcid.org/0000-0001-9113-8491 (Salih), https://orcid.org/0000-0003-1889-4690 (Duressa), https://orcid.org/0000-0003-2854-5080 (Ogra), \\ https://orcid.org/0000-0003-1033-3602 (Debela)
}

\begin{abstract}
This paper presents numerical solution of second order singularly perturbed self-adjoint boundary value problems using weighted residual method of Galerkin type. First, for the given problem, the residue was computed using appropriate approximated basis function which satisfies all the boundary conditions. Then, using the chosen weighting function, integrating the weighted residue over the domain and the given differential equation is transformed to linear systems of algebraic equations. Further, these algebraic equations were solved using Galerkin method. To validate the applicability of the proposed method, two model examples have been considered and solved for different values of perturbation parameter and with different order of basis function. Additionally, convergence of error bounds has been established for the method. As it can be observed from the numerical results, the present method approximates the exact solution very well. Moreover, the present method gives better accuracy when the order of basis function is increased and it also improves the result of the methods existing in the literature.
\end{abstract}

Keywords: Singularly perturbed problems, Self-adjoint problem, Galerkin method, Boundary value problems.

DOI: http://dx.doi.org/10.4314/ijest.v12i3.3

Cite this article as:

Salih M.H., Duressa G.F., Debela H.G. 2020. Numerical solution of singularly perturbed self-adjoint boundary value problem using Galerkin method. International Journal of Engineering, Science and Technology, Vol. 12, No. 3, pp. 26-37. doi: 10.4314/ijest.v12i3.3

Received: April 6, 2020; Accepted: May 8, 2020; Final acceptance in revised form: July 1, 2020

\section{Introduction}

Any differential equation obtained from a given differential equation and having the property that its solution is an integrating factor of the other is known as adjoint differential equation. If the coefficients $a_{0}(x), a_{1}(x)$ and $a_{2}(x)$ in the differential equation of the form:

$$
a_{0}(x) y^{\prime \prime}(x)+a_{1}(x) y^{\prime}(x)+a_{2}(x) y(x)=0
$$

are continuous and $a_{0}(x) \neq 0$ with the given domain, the obtained differential equation can be transformed into the equivalent self-adjoint equation of $\left(a(x) y^{\prime}(x)\right)^{\prime}+b(x) y(x)=0$ for the functions $a(x)=e^{\int \frac{a_{2}(x)}{a_{0}(x)} d x}$ and $b(x)=\frac{a_{2}(x)}{a_{0}(x)} a(x)$.

Singularly perturbed differential equation is a differential equation whose highest order derivative is multiplied by a small positive parameter. A self-adjoint differential equation, whose highest order derivative is multiplied by a small positive 
parameter, $\varepsilon(0<\varepsilon<<1)$, which has the form $-\varepsilon\left(a(x) y^{\prime}(x)\right)^{\prime}+b(x) y(x)=g(x)$ is called second order self-adjoint singular perturbation problem. A singular perturbation problem is a problem containing a small positive parameter that cannot be approximated by setting the parameter value to zero.

In singularly perturbed differential problem, small positive parameter affecting the highest order derivative(s) of the differential equation which gives rise to large gradients in the solution over narrow regions of the domain. The presence of a small perturbation parameter in the differential equation typically leads to boundary layers in the solution, which makes the convergence analysis very difficult (Yuzbas and Sahin, 2013). As Miller et al., (1996), Boundary layer is a region of the independent variable over which the dependent variable changes rapidly.

Singularly perturbed second order two-point boundary value problem occur very frequently in fluid motion, chemical reactor theory, elasticity, diffusion in polymer, reaction- diffusion equation, control of chaotic system and so on (Kadalbajoo and Kumar, 2008). If the order of singularly perturbed differential equations of the reduced problem is reduced by one then the problem called as convection-diffusion type and if the order is reduced by two it is called reaction-diffusion type. Hence, Second order singularly perturbed self-adjoint ordinary differential equations are types of reaction-diffusion problem. Due to the importance of these problems in real life situations, the need to develop numerical methods for approximating its solution is advantageous. But, numerically solving the singularly perturbed differential equations depends upon the small positive parameters. The solution varies rapidly in some parts of the domain and varies slowly in some other parts of the domain because of the existence of boundary layer. The solution of second order self-adjoint singularly perturbed two point boundary value problem exhibits one or two layers. For solving this problem having two layers, the existing numerical methods give good results when the mesh size $\mathrm{h}$ is smaller than the perturbation parameter $\mathcal{E}$ (i.e., $h \leq \mathcal{E}$ ). But it is expensive and time consuming process (Gelu et al., 2017). If we take $h \geq \mathcal{E}$, the existing numerical methods produce oscillatory solution and pollute the solution in the entire interval, because of boundary layer behavior. As a result, developing numerical methods for solving self-adjoint singular perturbation problems yield consideration of the researches.

Recently, different scholars like Gelu et al., (2016), Gelu et al., (2017) and Edosa and File (2017) have developed a higher (fourth, sixth, eighth and tenth) order compact finite difference method to solve singularly perturbed reaction diffusion problems. These authors' developed higher order compact finite difference methods, by considering the condition for the coefficients of diffusion and reaction terms are constant only. Thus, even if their methods produce more accurate numerical solution, it is restricted to treat the problems with constant coefficients of diffusion and reaction term. Similarly, Ali et al., (2018) have presented numerical study of self-adjoint singularly perturbed two-point boundary value problems using collocation method with error estimation and other scholars, Asrat et al., (2016) and Zeslassie et al., (2017) have presented fourth and sixth order stable central difference method for solving self-adjoint singularly perturbed two -point boundary value problem. As far as the researchers knowledge is concerned, numerical solution of second order self adjoint singularly perturbed boundary value problems via weight residual method of Galerkin type is first being considered and it has the following advantages: It works for both constant and variable coefficient, it is more accurate than the earlier method we listed in our literature and it is conceptually simple, easy to use and readily adaptable for computer implementation for solving singularly perturbed self-adjoint boundary value problems.

Therefore, the purpose of this paper is to formulate weighted residual method of Galerkin type by increasing the order of the basis function which gives more accurate results for solving second order self-adjoint singularly perturbed two point boundary value problems.

\section{Mathematical Formulation}

Consider the following self-adjoint singularly perturbed equation of the form:

$$
-\varepsilon \frac{d}{d x}\left(p(x) \frac{d y(x)}{d x}\right)+r(x) y(x)=z(x) \text { for } 0<x<1
$$

subject to the boundary conditions: for

$$
y(0)=\alpha, \quad y(1)=\beta
$$

where $\mathcal{E}(0<\varepsilon<<1)$, is a small positive perturbation parameter, $p(x), r(x)$ and $\mathrm{z}(x)$ are assumed to be sufficiently smooth known functions on $(0,1), \alpha$ and $\beta$ are known parameters.

For the derivation of the scheme, to make general let us consider our entire domain $\Omega=(a, b)$ in the form

$$
\begin{aligned}
y_{N} & =\sum_{j=1}^{N} c_{j} \phi_{j}(x)+\phi_{0}(x) \\
& =\phi_{0}(x)+c_{1} \phi_{1}+c_{2} \phi_{2}+\ldots+c_{N} \phi_{N}
\end{aligned}
$$


where $c_{j}$ are constant unknown parameter to be determined and $\phi_{j}(x)$ and $\phi_{0}(x)$ are basis functions chosen such that the specified boundary conditions of the problem are satisfied by the $N$ parameter approximate solution $y_{N}$. The particular form in Eq.(3) has two parts, one containing the unknowns $\sum_{j=1}^{N} c_{j} \phi_{j}$ which is called the homogeneous part and the other is the nonhomogeneous part $\left(\phi_{0}\right)$ satisfying the specified boundary conditions of the problem.

To apply the method, we choose the approximation basis function which satisfies the Eq. (2). For a choice of algebraic polynomials:

We assume $\phi_{0}(x)=c+d x$ and the two conditions on $\phi_{0}$ to determine the constant $c$ and $d$ we obtain

$$
\begin{aligned}
& \phi_{0}(a)=\alpha \Rightarrow c=\alpha-a d, \\
& \phi_{0}(b)=\beta \Rightarrow d=\frac{\beta-\alpha}{b-a} \\
& \phi_{0}(x)=c+d x=\alpha+\left(\frac{\beta-\alpha}{b-a}\right)(x-a),
\end{aligned}
$$

Therefore,

$$
\phi_{0}(x)=\alpha+\left(\frac{\beta-\alpha}{b-a}\right)(x-a)
$$

Similarly, choose $\phi_{j}$ which satisfies the boundary conditions in homogeneous form. Since there are two homogeneous conditions, we must assume at least a three parameter polynomial to obtain a nonzero function.

Let us assume $\phi_{1}(x)=m+n x+e x^{2}$, using the conditions on $\phi_{1}(x)$, we obtain:

$$
\begin{aligned}
& \phi_{1}(a)=0 \Rightarrow m=-a n-e a^{2} \\
& \phi_{1}(b)=0 \Rightarrow n=-e(b+a) \\
& \phi_{1}(x)=m+n x+e x^{2}=-e[(x-a)[(b+a)-(x+a)]]
\end{aligned}
$$

The constant $e$ can be set equal to unity because it will be absorbed in to the parameter notation $c_{1}$.

$$
\phi_{1}(x)=-[(x-a)[(b+a)-(x+a)]]
$$

For $\phi_{2}(x)$ we can assume one of the forms:

$$
\phi_{2}(x)=m+n x+t x^{3} \text { or } \phi_{2}(x)=m+e x^{2}+t x^{3}
$$

with $t \neq 0 ; \phi_{2}(x)$ does not contain all-order terms in either case, but the approximate solution is complete because $\left\{\phi_{1}, \phi_{2}\right\}$ contains all terms up to degree three.

Using conditions like $\phi_{1}$ for $\phi_{2}(x)=m+e x^{2}+t x^{3}$ we obtain:

$$
\begin{gathered}
\phi_{2}(a)=0 \Rightarrow m=-e a^{2}-t a^{3} \\
\phi_{2}(b)=0 \Rightarrow e=-t\left(\frac{b^{3}-a^{3}}{b^{2}-a^{2}}\right)
\end{gathered}
$$

Now, $\phi_{2}(x)=m+e x^{2}+t x^{3}=-t\left[\frac{a^{2}\left[a\left(b^{2}-a^{2}\right)-\left(b^{3}-a^{3}\right)\right]+x^{2}\left[\left(b^{3}-a^{3}\right)-x\left(b^{2}-a^{2}\right)\right]}{b^{2}-a^{2}}\right]$

The constant $t$ can be set equal to unity because it will be absorbed in to the parameter notation $c_{2}$.

Therefore,

$$
\phi_{2}(x)=-\left[\frac{a^{2}\left[a\left(b^{2}-a^{2}\right)-\left(b^{3}-a^{3}\right)\right]+x^{2}\left[\left(b^{3}-a^{3}\right)-x\left(b^{2}-a^{2}\right)\right]}{b^{2}-a^{2}}\right]
$$


In the same way, choose $\phi_{3}(x)$ which satisfies all the boundary conditions in homogeneous form.

For $\phi_{3}(x)$ we can assume one of the forms:

$$
\phi_{3}(x)=m+n x+g x^{4} \text { or } \phi_{3}(x)=m+e x^{2}+g x^{4} \text { or } \phi_{3}(x)=m+t x^{3}+g x^{4} .
$$

Using the conditions on $\phi_{1}(x)$ and $\phi_{2}(x)$ for $\phi_{3}(x)=m+t x^{3}+g x^{4}$, we obtain;

$$
\begin{aligned}
& \phi_{3}(a)=0 \Rightarrow m=-t a^{3}-g a^{4} \\
& \phi_{3}(b)=0 \Rightarrow t=-g\left(\frac{b^{4}-a^{4}}{b^{3}-a^{3}}\right)
\end{aligned}
$$

Now,

$$
\begin{aligned}
\phi_{3}(x)=m+t x^{3}+g x^{4}=a^{3}\left(g\left(\frac{b^{4}-a^{4}}{b^{3}-a^{3}}\right)\right)-g a^{4}-g x^{3}\left(\frac{b^{4}-a^{4}}{b^{3}-a^{3}}\right)+g x^{4} \\
\phi_{3}(x)=-g\left[\frac{a^{3}\left[a\left(b^{3}-a^{3}\right)-\left(b^{4}-a^{4}\right)\right]+x^{3}\left[\left(b^{4}-a^{4}\right)-x\left(b^{3}-a^{3}\right)\right]}{b^{3}-a^{3}}\right]
\end{aligned}
$$

The constant $g$ can be set equal to unity because it will be absorbed in to the parameter notation $c_{3}$ Therefore, for the third choice

$\phi_{3}(x)$ we obtain; $\quad \phi_{3}(x)=-\left[\frac{a^{3}\left[a\left(b^{3}-a^{3}\right)-\left(b^{4}-a^{4}\right)\right]+x^{3}\left[\left(b^{4}-a^{4}\right)-x\left(b^{3}-a^{3}\right)\right]}{b^{3}-a^{3}}\right]$

In generally, the chosen $\phi_{1}, \phi_{2}$ and $\phi_{3}$ for, $(j=1,2 \ldots, N) \phi_{j}$ is given as follows

$$
\begin{gathered}
\phi_{1}(x)=-[(x-a)[(b+a)-(x+a)]] \\
\phi_{2}(x)=-\left[\frac{a^{2}\left[a\left(b^{2}-a^{2}\right)-\left(b^{3}-a^{3}\right)\right]+x^{2}\left[\left(b^{3}-a^{3}\right)-x\left(b^{2}-a^{2}\right)\right]}{b^{2}-a^{2}}\right] \\
\phi_{3}(x)=-\left[\frac{a^{3}\left[a\left(b^{3}-a^{3}\right)-\left(b^{4}-a^{4}\right)\right]+x^{3}\left[\left(b^{4}-a^{4}\right)-x\left(b^{3}-a^{3}\right)\right]}{b^{3}-a^{3}}\right] \\
\vdots \\
\phi_{N}(x)=-\left[\frac{a^{N}\left[a\left(b^{N}-a^{N}\right)-\left(b^{N+1}-a^{N+1}\right)\right]+x^{N}\left[\left(b^{N+1}-a^{N+1}\right)-x\left(b^{N}-a^{N}\right)\right]}{b^{N}-a^{N}}\right]
\end{gathered}
$$

for $N$ is a positive integer.

We choose a set of basis functions $\left\{\phi_{j}, j=1,2, \ldots, N\right\}$, and make an approximation of the form of Eq. (3)

$$
y_{N}=\sum_{j=1}^{N} c_{j} \phi_{j}(x)+\phi_{0}(x)
$$

The basis functions can be polynomials functions, trigonometric functions or other functions. But in our case the basis functions are polynomial because polynomial functions are continuous, easily differentiable, integrable and suitable for programming. Weighted integral method (WIM) is a class of method used to obtain the approximate solution to the differential equations in matrix form as

$$
A y=z, \text { in } \Omega \text {. }
$$

where $A$ is an operator (linear or nonlinear), often a differential operator, acting on the dependent variable $y$, and $z$ is a known function of the independent variables.

To apply the WRM, we can approximate $y(x)$ of the differential equation in Eq. (8) in to $y_{N}(x)$. When $y_{N}(x)$ is substituted into Eq. (8), it is unlikely that the equation is satisfied.

$$
\Rightarrow A y_{N}(x)-z=A\left(\sum_{j=1}^{N} c_{j} \phi_{j}(x)+\phi_{0}(x)\right)-z \neq 0
$$




$$
\Rightarrow R \equiv A y_{N}(x)-z
$$

where $R$ is residual of the approximation.

Multiply Eq. (9) by an arbitrary weighted function $w(x)$ and integrating over the domain $\Omega$ to force this integral to vanish over the given domain to obtain the unknown parameters:

$$
\int_{\Omega} w(x) R(x) d \Omega=0
$$

In the weighted residual method, the unknown parameters $c_{j}$ 's are determined by minimize the residual $R$ in some special cases. Different methods of minimizing the residual yield different approximate solutions.

When the weight functions are chosen to be the basis functions themselves, then it is known as Galerkin method. We set

$$
\phi_{i}(x)=w_{i}(x), \quad \mathrm{i}=1,2, \ldots, N
$$

The unknown coefficients in the approximate solution are determined by setting the integral over $\Omega$ of the weighted residual to zero.

For one-dimensional problem in the interval $(a, b)$, this procedure will result:

$$
\int_{a}^{b} \phi_{i}(x) R(x) d x=\int_{a}^{b} w_{i}(x) R(x) d x=0, \quad(\mathrm{i}=1,2, \ldots, N)
$$

The present method considers result in a system of equations of the form:

$$
\begin{aligned}
& \int_{\Omega}\left(\phi_{i}\left[A \phi_{0}+\sum_{j=1}^{N} c_{j} A \phi_{j}-z\right]\right) d x=0 \\
& c_{j} \int_{\Omega} \phi_{i} A \phi_{j} d x=\int_{\Omega} \phi_{i}\left[z-A \phi_{0}\right] d \Omega \\
& D c=B
\end{aligned}
$$

where $D_{i j}=\int_{\Omega} \phi_{i} A \phi_{j} d x, B_{i}=\int_{\Omega} \phi_{i}\left[z-A \phi_{0}\right] d x, D$ is matrices and $c$ is unknown vectors and $B$ is a column vector.

\section{Convergence of the method}

Consider the following self-adjoint singularly perturbed equation of the form:

$$
-\varepsilon \frac{d}{d x}\left(p(x) \frac{d y(x)}{d x}\right)+r(x) y(x)=z(x) \quad \text { for } a<x<b
$$

subject to the boundary conditions:

The weak form of the above problem is:

$$
y(a)=\alpha, \quad y(b)=\beta
$$

$$
\int_{a}^{b} w\left(-\varepsilon \frac{d}{d x}\left(p(x) \frac{d y(x)}{d x}\right)\right) d x+\int_{a}^{b} w r(x) y(x) d x-\int_{a}^{b} w z(x) d x
$$

By using integration by part

$$
\int_{a}^{b} w\left(-\varepsilon \frac{d}{d x}\left(p(x) \frac{d y(x)}{d x}\right)\right) d x=\int_{a}^{b} \varepsilon p \frac{d y}{d x} \frac{d w}{d x} d x-\left.\left(\varepsilon p w \frac{d y}{d x}\right)\right|_{a} ^{b}
$$

Now substituting Eq. (14) in to Eq. (13) we

$$
\begin{aligned}
& \text { get } \int_{a}^{b} w\left(-\varepsilon \frac{d}{d x}\left(p(x) \frac{d y(x)}{d x}\right)\right) d x+\int_{a}^{b} w r(x) y(x) d x-\int_{a}^{b} w z(x) d x=\int_{a}^{b}\left(\varepsilon p \frac{d y}{d x} \frac{d w}{d x}+w r(x) y(x)\right) d x \\
& -\int_{a}^{b} w z(x) d x-\left.\left(\varepsilon p w \frac{d y}{d x}\right)\right|_{a} ^{b}
\end{aligned}
$$


Since the give boundary condition Dirichlet boundary condition assume that $y(a)=y(b)=0$. Therefore $\left.\left(\varepsilon p w \frac{d y}{d x}\right)\right|_{a} ^{b}=0$

$$
\begin{aligned}
\int_{a}^{b} w\left(-\varepsilon \frac{d}{d x}\left(p(x) \frac{d y(x)}{d x}\right)\right) d x & +\int_{a}^{b} w r(x) y(x) d x-\int_{a}^{b} w z(x) d x \\
= & \int_{a}^{b}\left(\varepsilon p \frac{d y}{d x} \frac{d w}{d x}+w r(x) y(x)\right) d x-\int_{a}^{b} w z(x) d x \\
\int_{a}^{b} w\left(-\varepsilon \frac{d}{d x}\left(p(x) \frac{d y(x)}{d x}\right)\right) d x & +\int_{a}^{b} w r(x) y(x) d x-\int_{a}^{b} w z(x) d x=B(y, w)-l(w)
\end{aligned}
$$

The quadratic functional of the Eq. (15) is:

$$
\begin{aligned}
& I(y)=\frac{1}{2} B(y, y)-l(y), \text { since } y=w \text { then } \\
& I(y)=\int_{a}^{b} \frac{1}{2}\left(\varepsilon p \frac{d y}{d x} \frac{d y}{d x}+y r y\right) d x-\int_{a}^{b} y z d x \\
&=\int_{a}^{b} \frac{1}{2}\left(\varepsilon p\left(\frac{d y}{d x}\right)^{2}+y^{2} r\right) d x-\int_{a}^{b} y z d x \\
& I(y)=\int_{a}^{b} \frac{1}{2}\left(\varepsilon p\left(\frac{d y}{d x}\right)^{2}+y^{2} r-2 y z\right) d x
\end{aligned}
$$

Similarly,

$$
I\left(y_{N}\right)=\int_{a}^{b} \frac{1}{2}\left(\varepsilon p\left(\frac{d y_{N}}{d x}\right)^{2}+y_{N}^{2} r-2 y_{N} z\right) d x
$$

Now $\quad I\left(y_{N}\right)-I(y)=\int_{a}^{b} \frac{1}{2}\left(\varepsilon p\left(\frac{d y_{N}}{d x}\right)^{2}+y_{N}{ }^{2} r-2 y_{N} z\right) d x-\left(\int_{a}^{b} \frac{1}{2}\left(\varepsilon p\left(\frac{d y}{d x}\right)^{2}+y^{2} r-2 y z\right) d x\right)$

$$
\begin{gathered}
=\int_{a}^{b} \frac{1}{2}\left(\varepsilon p\left(\frac{d y_{N}}{d x}\right)^{2}+y_{N}{ }^{2} r-2 y_{N} z-\varepsilon p\left(\frac{d y}{d x}\right)^{2}-y^{2} r+2 y z\right) d x \\
I\left(y_{N}\right)-I(y)=\int_{a}^{b} \frac{1}{2}\left(\varepsilon p\left(\frac{d y_{N}}{d x}\right)^{2}-\varepsilon p\left(\frac{d y}{d x}\right)^{2}+y_{N}{ }^{2} r-y^{2} r\right) d x+\int_{a}^{b} z\left(y-y_{N}\right) d x
\end{gathered}
$$

since $z(x)=-\varepsilon \frac{d}{d x}\left(p(x) \frac{d y(x)}{d x}\right)+r(x) y(x)$

$$
\begin{aligned}
\int_{a}^{b} z\left(y-y_{N}\right) d x & =\int_{a}^{b}\left(-\varepsilon \frac{d}{d x}\left(p \frac{d y}{d x}\right)+r y\right)\left(y-y_{N}\right) d x \\
& =\int_{a}^{b}\left(\varepsilon p \frac{d y}{d x} \frac{d}{d x}\left(y-y_{N}\right)+r y\left(y-y_{N}\right)\right) d x-\left.\left(\varepsilon p\left(y-y_{N}\right) \frac{d y}{d x}\right)\right|_{a} ^{b}
\end{aligned}
$$

Substitute Eq. (19) in to Eq. (18) we get: 


$$
\begin{aligned}
\int_{a}^{b} z\left(y-y_{N}\right) d x & =\int_{a}^{b} \frac{1}{2}\left(\varepsilon p\left(\frac{d y_{N}}{d x}\right)^{2}-\varepsilon p\left(\frac{d y}{d x}\right)^{2}+y_{N}{ }^{2} r-y^{2} r\right) d x \\
& +\int_{a}^{b}\left(\varepsilon p \frac{d y}{d x} \frac{d}{d x}\left(y-y_{N}\right)+r y\left(y-y_{N}\right)\right) d x-\left.\left(\varepsilon p\left(y-y_{N}\right) \frac{d y}{d x}\right)\right|_{a} ^{b} \\
& =\int_{a}^{b}\left(\frac{1}{2} \varepsilon p\left(\frac{d y_{N}}{d x}\right)^{2}-\frac{1}{2} \varepsilon p\left(\frac{d y}{d x}\right)^{2}+\frac{1}{2} y_{N}^{2} r-\frac{1}{2} y^{2} r+\varepsilon p\left(\frac{d y}{d x}\right)^{2}-\varepsilon p \frac{d y}{d x} \frac{d y_{N}}{d x}+r y^{2}-r y y_{N}\right) d x \\
& =\int_{a}^{b}\left(\frac{1}{2} \varepsilon p\left(\frac{d y_{N}}{d x}\right)^{2}+\frac{1}{2} \varepsilon p\left(\frac{d y}{d x}\right)^{2}+\frac{1}{2} y_{N}{ }^{2} r+\frac{1}{2} y^{2} r-\varepsilon p \frac{d y}{d x} \frac{d y_{N}}{d x}-r y y_{N}\right) d x \\
& =\int_{a}^{b} \frac{1}{2}\left(\varepsilon p\left(\frac{d y_{N}}{d x}\right)^{2}-2 \varepsilon p \frac{d y}{d x} \frac{d y_{N}}{d x}+\varepsilon p\left(\frac{d y}{d x}\right)^{2}+r\left(y_{N}^{2}-2 y y_{N}+y^{2}\right)\right) d x \\
& =\int_{a}^{b}\left(\frac{1}{2} \varepsilon p\left(\frac{d y_{N}}{d x}-\frac{d y}{d x}\right)^{2}+\frac{1}{2} r\left(y_{N}-y\right)^{2}\right) d x \geq 0 \\
& \Rightarrow I\left(y_{N}\right)-I(y) \geq 0,
\end{aligned}
$$

So,

$$
I\left(y_{N}\right) \geq I(y)
$$

Therefore, convergence of energy of the approximate solution to the exact solution is from the above and the exact solution minimizes the energy. The data of the problem is sufficiently continues, differentiable and integrable. This guarantees convergence of the method.

\section{Illustrative Examples and Results}

To demonstrate the applicability of the methods, two model self-adjoint singularly perturbed problems have been considered. These examples have been chosen because they have been widely discussed in the literature and their exact solutions were available for comparison.

Example 1: Consider the singularly perturbed problem:

$$
-\varepsilon\left(\left(1+x^{2}\right) y^{\prime}\right) y^{\prime}+\left(1+x-x^{2}\right) y=f(x), \quad 0<x<1 \quad \text { with } \quad y(0)=y(1)=0
$$

where $z(x)=1+x(1-x)-e^{\left(\frac{-x}{\sqrt{\varepsilon}}\right)}\left[x\left(2 x^{2}-3 x+1\right)-\left(2 \sqrt{\varepsilon}\left(2 x^{2}-x(1+\sqrt{\varepsilon})+1\right)\right]\right.$

$$
+e^{-\left(\frac{1-x}{\sqrt{\varepsilon}}\right)}\left[x^{2}(2 x-1)+\left(2 \sqrt{\varepsilon}\left(2 x^{2}+x \sqrt{\varepsilon}+1\right)\right]\right.
$$

The exact solution is given by:

$$
y(x, \varepsilon)=1+(x-1) e^{\left(\frac{-x}{\sqrt{\varepsilon}}\right)}-x e^{-\left(\frac{1-x}{\sqrt{\varepsilon}}\right)}
$$

The numerical solutions in terms of maximum absolute errors are given in Tables 1-2 and Figures 1-2.

Table 1: Maximum absolute errors of Example 1 for different order of base function

\begin{tabular}{lcccc}
\hline $\mathcal{E}$ & Order 3 & Order 5 & Order 7 & Order 9 \\
\hline $2^{0}$ & $8.1767 \times 10^{-6}$ & $1.0615 \times 10^{-8}$ & $2.8640 \times 10^{-7}$ & $8.6945 \times 10^{-4}$ \\
$2^{-3}$ & $7.0081 \times 10^{-4}$ & $7.8164 \times 10^{-6}$ & $5.2035 \times 10^{-8}$ & $5.8425 \times 10^{-8}$ \\
$2^{-5}$ & $7.5765 \times 10^{-3}$ & $3.1996 \times 10^{-4}$ & $8.1669 \times 10^{-6}$ & $5.4286 \times 10^{-7}$ \\
$2^{-7}$ & $4.8755 \times 10^{-2}$ & $5.6437 \times 10^{-3}$ & $4.8615 \times 10^{-4}$ & $3.0480 \times 10^{-5}$ \\
$2^{-9}$ & $1.8217 \times 10^{-1}$ & $4.9736 \times 10^{-2}$ & $8.9656 \times 10^{-3}$ & $1.6241 \times 10^{-3}$ \\
$2^{-11}$ & $3.9651 \times 10^{-1}$ & $1.8364 \times 10^{-1}$ & $4.8905 \times 10^{-2}$ & $2.1081 \times 10^{-2}$ \\
\hline
\end{tabular}


Table 2: Maximum absolute errors for Example 1for $N=16$ (using ninth order base function)

\begin{tabular}{ccc}
\hline $\mathcal{E}$ & Present method & Asrat et al.,2016 \\
\hline $2^{-4}$ & $5.9456 \times 10^{-8}$ & $5.8500 \times 10^{-4}$ \\
$2^{-5}$ & $5.4286 \times 10^{-7}$ & $8.5700 \times 10^{-4}$ \\
$2^{-6}$ & $2.5381 \times 10^{-6}$ & $9.5800 \times 10^{-4}$ \\
$2^{-7}$ & $3.0480 \times 10^{-5}$ & $1.2900 \times 10^{-3}$ \\
$2^{-8}$ & $2.6089 \times 10^{-4}$ & $1.6500 \times 10^{-3}$ \\
$2^{-12}$ & $4.3854 \times 10^{-2}$ & $3.7600 \times 10^{-2}$ \\
\hline
\end{tabular}

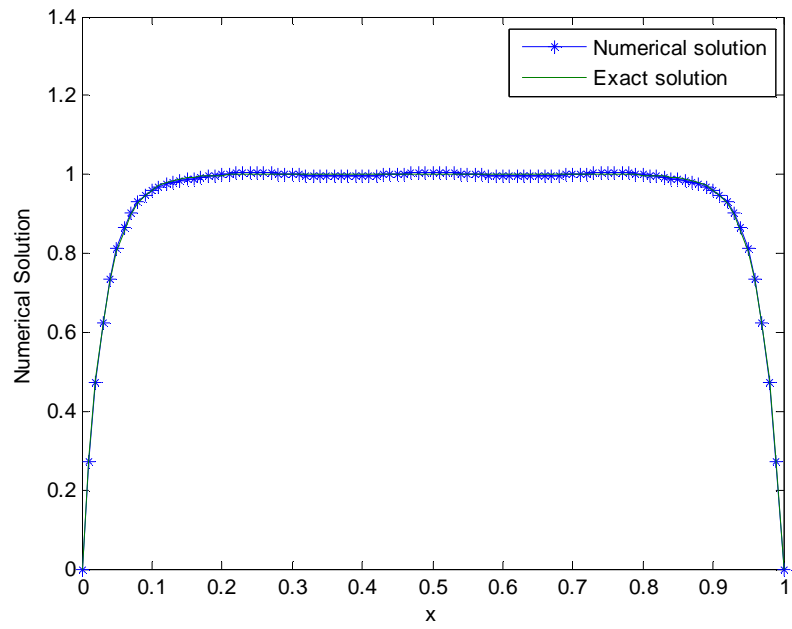

Figure 1: The behavior of exact and numerical solution for Example 1 at $\varepsilon=10^{-3}$ and $N=100$

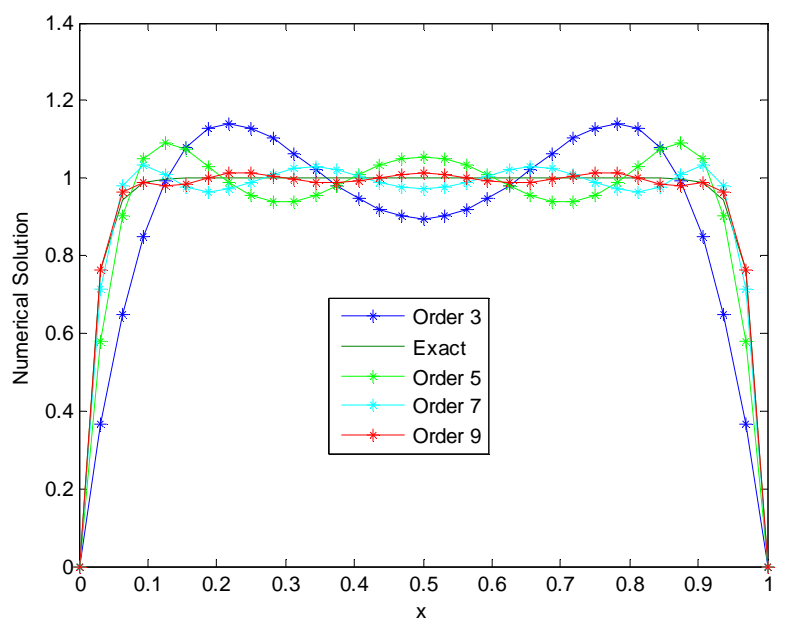

Figure 2: Numerical solution of Example 1of different order of base function when $\mathcal{E}=2^{-11}$

$$
\text { and } N=32 \text {. }
$$




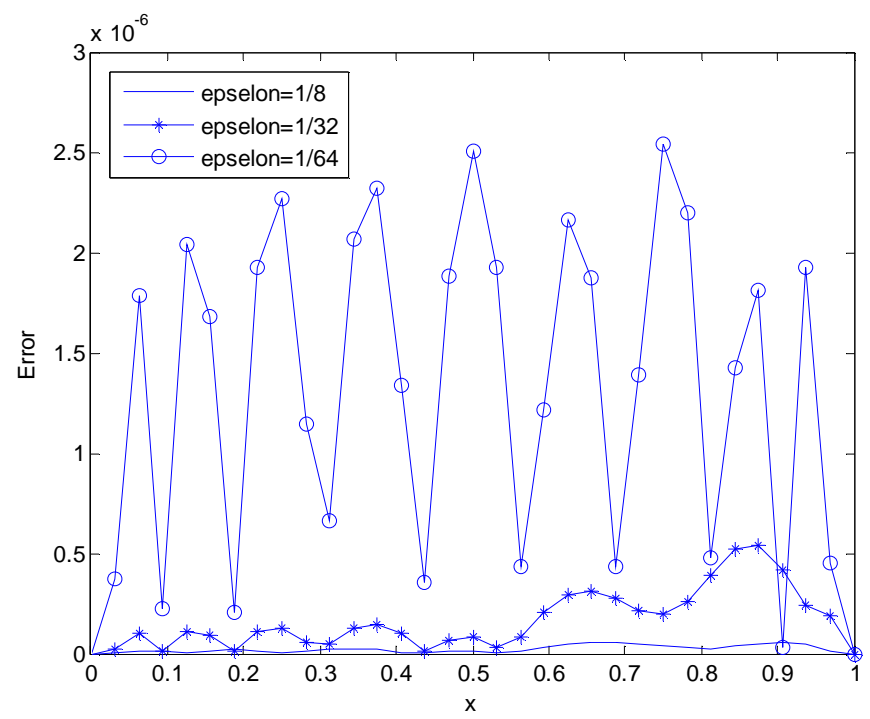

Figure 3: Point wise absolute errors for Example 1 at $N=32$ and different perturbation parameters.

Example 2: Consider the singularly perturbed problem:

$$
-\varepsilon y^{\prime \prime}+\left(1-x-x^{2}\right) y=f(x), \quad 0<x<1 \quad \text { with } \quad y(0)=y(1)=0
$$

where $z(x)=1+x-x^{2}+\left(2 \sqrt{\varepsilon}-x^{2}+x^{3}\right) e^{-\left(\frac{1-x}{\sqrt{\varepsilon}}\right)}+\left(2 \sqrt{\varepsilon}-x(1-x)^{2}\right) e^{\left(\frac{-x}{\sqrt{\varepsilon}}\right)}$

The exact solution is given by:

$$
y(x, \varepsilon)=1+(x-1) e^{\left(\frac{-x}{\sqrt{\varepsilon}}\right)}-x e^{-\left(\frac{1-x}{\sqrt{\varepsilon}}\right)}
$$

The numerical solutions in terms of maximum absolute errors are given in Tables 3-5 and figures 3-4.

Table 3: Maximum absolute errors of Example 2 for different order of base function

\begin{tabular}{ccccc}
\hline $\mathcal{E}$ & Order 3 & Order 5 & Order 7 & Order 9 \\
\hline $2^{-2}$ & $1.7321 \times 10^{-4}$ & $9.7792 \times 10^{-7}$ & $3.4644 \times 10^{-9}$ & $3.1349 \times 10^{-7}$ \\
$2^{-4}$ & $2.4999 \times 10^{-3}$ & $5.4650 \times 10^{-5}$ & $7.1434 \times 10^{-7}$ & $5.9456 \times 10^{-8}$ \\
$2^{-6}$ & $1.9313 \times 10^{-2}$ & $1.4944 \times 10^{-3}$ & $7.3215 \times 10^{-5}$ & $2.5381 \times 10^{-6}$ \\
$2^{-8}$ & $1.0077 \times 10^{-1}$ & $1.7969 \times 10^{-2}$ & $2.4117 \times 10^{-3}$ & $2.6089 \times 10^{-4}$ \\
$2^{-10}$ & $2.8574 \times 10^{-1}$ & $1.0700 \times 10^{-1}$ & $2.3994 \times 10^{-2}$ & $7.1512 \times 10^{-3}$ \\
$2^{-12}$ & $4.9550 \times 10^{-1}$ & $2.6125 \times 10^{-1}$ & $8.6681 \times 10^{-2}$ & $4.3854 \times 10^{-2}$ \\
\hline
\end{tabular}

Table 4: Maximum absolute errors for Example 2 for $N=16$ (using ninth order base function)

\begin{tabular}{lll}
\hline $\mathcal{E}$ & Present method & Asrat et al., 2016 \\
\hline $1 / 8$ & $5.9456 \times 10^{-8}$ & $1.424 \times 10^{-6}$ \\
$1 / 16$ & $5.9456 \times 10^{-8}$ & $4.148 \times 10^{-6}$ \\
$1 / 32$ & $5.4286 \times 10^{-7}$ & $9.622 \times 10^{-6}$ \\
$1 / 64$ & $2.5381 \times 10^{-6}$ & $3.074 \times 10^{-4}$ \\
$1 / 128$ & $3.0480 \times 10^{-6}$ & $1.301 \times 10^{-4}$ \\
$1 / 256$ & $2.6089 \times 10^{-4}$ & $5.910 \times 10^{-4}$ \\
\hline
\end{tabular}


Table 5: Maximum absolute errors for Example 2 for $N=32$ (using ninth order base function)

\begin{tabular}{lcl}
\hline \multicolumn{1}{c}{$\varepsilon$} & Present method & Ali et al., 2018 \\
\hline 1 & $8.3600 \times 10^{-8}$ & $3.9200 \times 10^{-9}$ \\
$1 / 10$ & $7.9975 \times 10^{-7}$ & $8.8600 \times 10^{-7}$ \\
$1 / 100$ & $1.2940 \times 10^{-5}$ & $1.7700 \times 10^{-5}$ \\
$1 / 1000$ & $6.8420 \times 10^{-3}$ & $4.0500 \times 10^{-4}$ \\
\hline
\end{tabular}

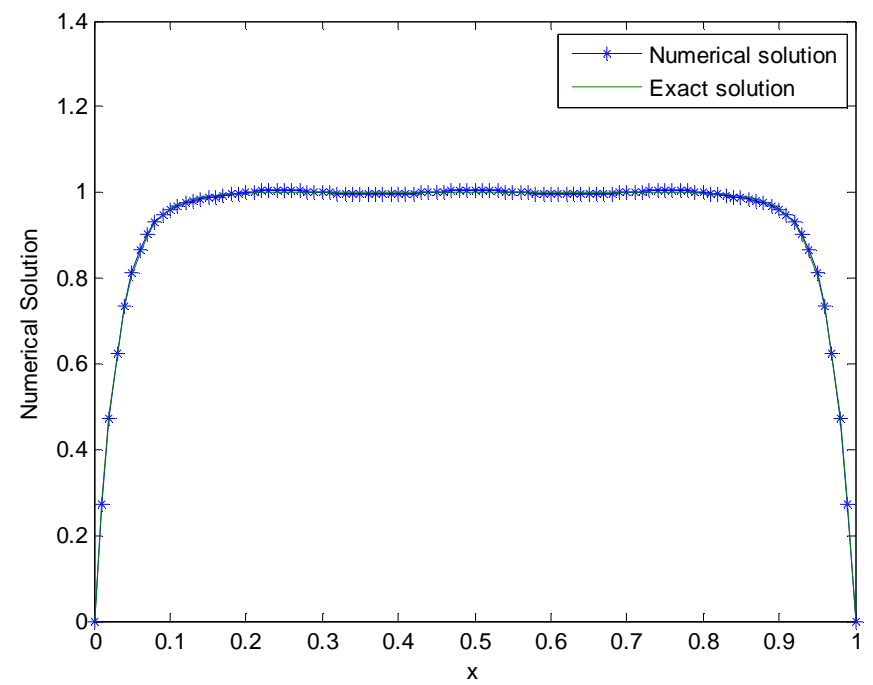

Figure 4: The behavior of exact and numerical solution for Example 2 at $\varepsilon=10^{-3}$ and $N=100$

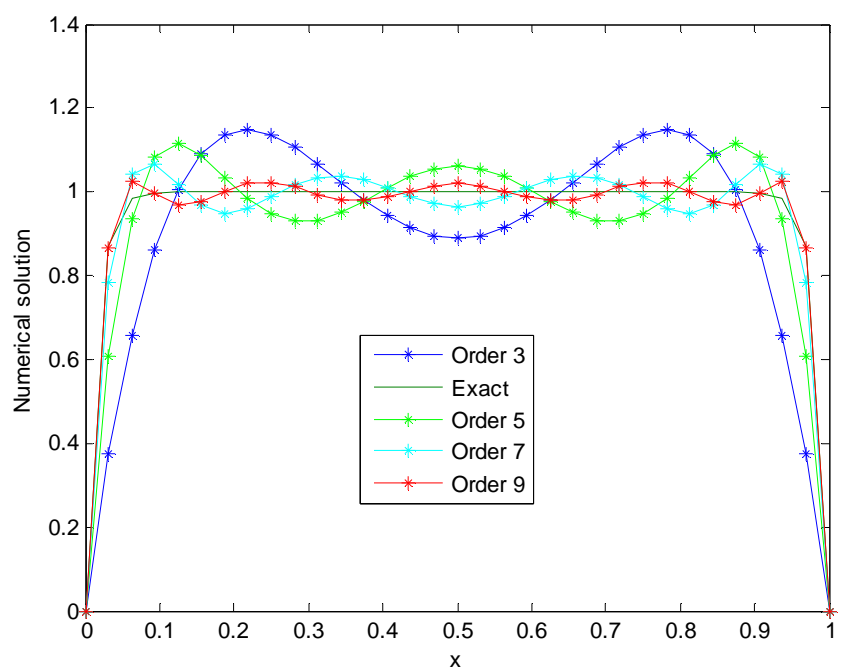

Figure 5: Numerical solution of Example 1of different order of base function when $\mathcal{E}=2^{-11}$ and $N=32$. 


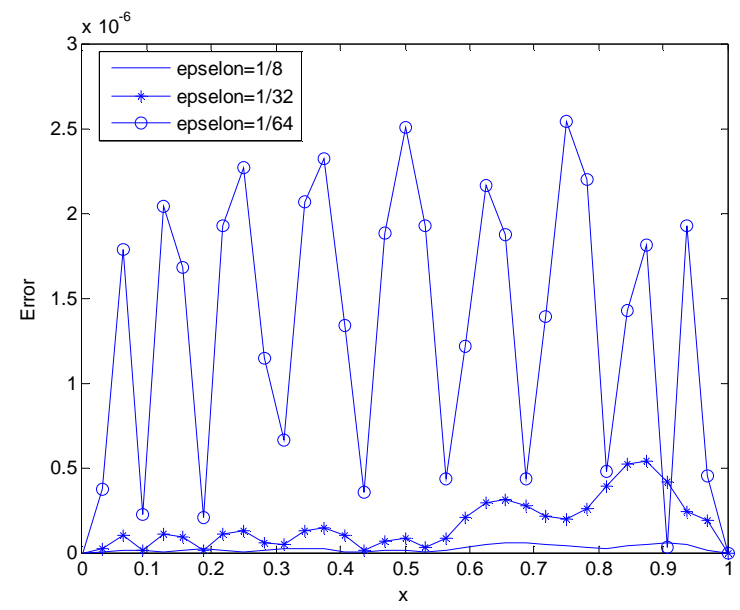

Figure 6: Point wise absolute errors for Example 1 at $N=32$ and different perturbation parameters.

\section{Discussion}

This study introduces weighted residual method of Galerkin type for solving second order self-adjoint singularly perturbed boundary value problems. To demonstrate the competence of the method, we realized two model examples by taking different values for the perturbation parameter, $\varepsilon$, and mesh size, $h$. The numerical results obtained by the present method have been compared with the numerical results presented by more recent authors like, Asrat et al., (2016) and Ali et al., (2018); and it is observed that the present method gives more accurate results than some findings reported in literatures. The numerical results are tabulated in terms of maximum absolute errors (see Tables 1-5) and compared with the results of the previously developed numerical methods existing in the literature (Table 2, 4, 5). Further, behavior of the numerical solution with exact solution (Figure 1 and 4), behavior of the numerical solution with different order of basis function (Figure 2 and 5), point-wise absolute errors (Figure 3 and 6 ) are plotted graphically. In concise manner, the present methods are conceptually simple, easy to use and readily adaptable for computer implementation for solving singularly perturbed self-adjoint boundary value problems.

\section{Conclusion}

This paper presents numerical solution of second order singularly perturbed self-adjoint boundary value problems using weighted residual method of Galerkin type. The convergence analysis is investigated. As the formulated scheme is validated by numerical model examples and results, one can realize that the maximum absolute error decreases as mesh size $h$ decreases, which in turn shows the convergence of the computed solution. Furthermore, the result of the present method is compared with previous findings and shows that, it is more accurate than some existing numerical methods reported in the literature and approximates the exact solution very well. Generally, the present method is stable, and gives more accurate numerical solution for solving second order self-adjoint singularly perturbed boundary value problems.

\section{Acknowledgement}

The authors would like to thank the College of Natural Sciences, Jimma Universities for financial and material support.

\section{References}

Ali K.K., Hadhoud A.R., Shaalan M.A. 2018. Numerical study of self-adjoint singularly perturbed two-point boundary value problems using collocation method with error estimation. Journal of Ocean Engineering and Science. Vol. 3, No. 3, pp. 237243. https://doi.org/10.1016/j.joes.2018.07.001

Asrat T., File G. and Aga T. 2016. Fourth-order stable central difference method for self-adjoint singular perturbation problems, Ethiopian Journal of Science and Technology, Vol. 9, No. 1, pp. 53-68. DOI: 10.4314/ejst.v9i1.5

Edosa F. and File G. 2017. Higher order compact finite difference method for singularly perturbed one dimensional reaction diffusion problems, Journal of the Nigerian Mathematical Society, Vol. 36, No. 3, pp. 491-502.

Galu F.W., Duressa G.F. and Bullo T.A. 2016. Tenth order compact finite difference method for solving singularly perturbed 1D reaction - diffusion equations, International Journal of Engineering and Applied Sciences, Vol.8, pp. 15-24. 
Gelu F.W., Duressa G.F. and Bullo T.A. 2017. Sixth-order compact finite difference method for singularly perturbed 1D reaction diffusion problems, Journal of Taibah University for Science, Vol.11, No. 2, pp. 302-308. https://doi.org/10.1016/j.jtusci.2015.12.010

Kadalbajoo M. K. and Kumar D. 2008. Parameter-uniform fitted operator B-spline collocation method for self-adjoint singularly perturbed two-point boundary value problems, Electronic Transactions on Numerical Analysis. Vol. 30, 346-358.

Miller J.J.H, O'Riordan E and Shishkin G.I. 1996. Fitted numerical methods for singular perturbation problems, Error estimate in the maximum norm for linear problems in one and two dimensions, World Scientific. ISBN: 981-02-2462-1.

Yuzbas S. and Sahin N. 2013. Numerical solutions of singularly perturbed one-dimensional parabolic convection-diffusion problems by the Bessel collocation method, Applied Mathematics and Computation. Vol. 220, pp. 305-315. https://doi.org/10.1016/j.amc.2013.06.027

Zeslassie Y., File G. and Aga T. 2017. Sixth-order stable central difference method for self-adjoint singular perturbation problems, Ethiopian Journal of Education and Sciences, Vol. 13, No. 1, pp. 23-41.

\section{Biographical notes}

Murad Hussein Salih received MSc in mathematics (Numerical Analysis) from Jimma University, Ethiopia in 2018.

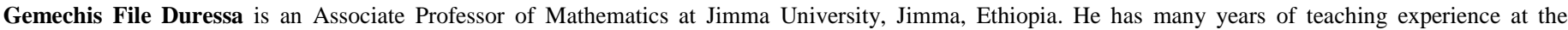
university level. Currently he is working as Dean of College of Natural sciences in Jimma University. He has more than 40 peer-reviewed publications.

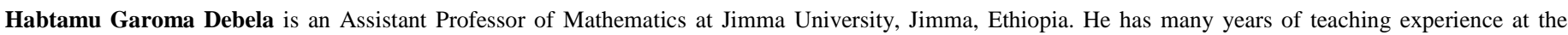
university level. Currently, he is a PhD candidate at Jimma University, Ethiopia. He has published more than 9 peer-reviewed publications in international journals. 